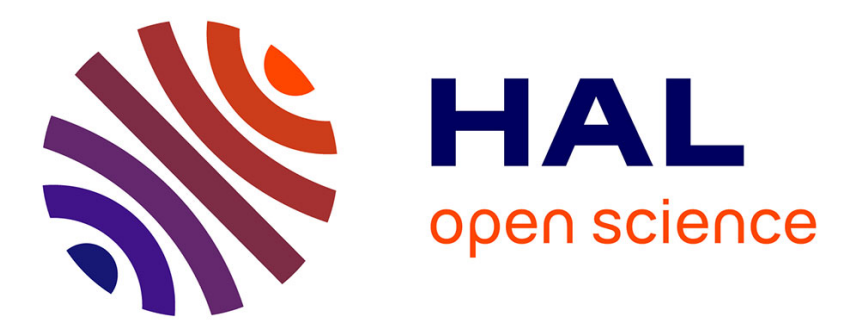

\title{
Séparations de phases dans les solutions solides métalliques
}

F. Bley, J. Desplat, P. Guyot, F. Livet, J. Mainville, J. Simon

\section{To cite this version:}

F. Bley, J. Desplat, P. Guyot, F. Livet, J. Mainville, et al.. Séparations de phases dans les solutions solides métalliques. Journal de Physique IV Proceedings, 1994, 04 (C3), pp.C3-15-C3-24. 10.1051/jp4:1994302 . jpa-00252499

\section{HAL Id: jpa-00252499 https://hal.science/jpa-00252499}

Submitted on 1 Jan 1994

HAL is a multi-disciplinary open access archive for the deposit and dissemination of scientific research documents, whether they are published or not. The documents may come from teaching and research institutions in France or abroad, or from public or private research centers.
L'archive ouverte pluridisciplinaire HAL, est destinée au dépôt et à la diffusion de documents scientifiques de niveau recherche, publiés ou non, émanant des établissements d'enseignement et de recherche français ou étrangers, des laboratoires publics ou privés. 


\title{
Séparations de phases dans les solutions solides métalliques
}

\author{
F. BLEY, J. DESPLAT, P. GUYOT, F. LIVET, J. MAINVILLE et J.P. SIMON
}

LTPCM-ENSEEG, URA 029 du CNRS, BP. 75, 38402 Saint Martin d'Hères cedex, France

\begin{abstract}
A number of metallic alloys display phase separation between two coherent phases. This paper will describe how to characterize the dynamic evolution of a metallic solid solution quenched inside a miscibility gap. The combination of various measurement techniques allows one to obtain complementary informations on the progress of unmixing; their interpretation will be discussed in the light of theories. The kinetics regime at the onset of unmixing depends on the depth of the quench and on the distance to the critical point : it ranges from nucleation-growth for shallow quenches away from the critical point to spinodal decomposition for deep quenches or quenches near the critical point. In the coarsening regime, the phase morphology will affect the growth kinetics. The morphology will depend on properties of the phases themselves (eg. : ordered phase), on the volume fractions, on size effects which introduce an anisotropy and modify the location of the coherent miscibility gap.
\end{abstract}

\section{Introduction}

La démixtion est un phénomène qui joue un grand rôle en métallurgie ; elle contrôle un bon nombre de propriétés des alliages, en particulier le durcissement tant du point de vue mécanique qu'électrique ou magnétique.

Nous traitons ici de la cinétique de retour à l'équilibre d'un alliage trempé brusquement d'une température $\mathrm{T}_{\mathrm{a}}$ où il est en solution solide, à une température $\mathrm{T}_{\mathrm{r}}$ située sous la lacune de miscibilité, où l'état d'équilibre est biphasé. Nous nous intéressons au cas d'une démixtion continue, c'est à dire se produisant dans la masse du matériau et non sur des défauts, et cohérente, c'est à dire où les effets élastiques sont suffisamment faibles pour qu'il n'y ait pas de défaut d'interface. Les théories de la démixtion traitent en premier lieu du cas d'une démixtion de type purement chimique, les structures cristallines des deux phases étant identiques. Les effets élastiques, liếs à la différence de taille des atomes, sont rajoutés, si nécessaire, dans l'équation cinétique. Ces théories peuvent être étendues au cas où l'une des phases est ordonnée. Le mécanisme qui permet cette démixtion est la diffusion des atomes par migration de lacunes.

\section{Théorie - Mécanismes}

\section{Généralités}

Une lacune de miscibilité, de type purement chimique est schématisée sur la figure 1a. Elle représente une transition de phase du premier ordre, sauf au point critique $\left(c_{c}, T_{c}\right)$, où elle est du second ordre. Au dessus de cette lacune, l'alliage à l'équilibre présente des fluctuations de concentration dynamiques, qu'on peut appeler "ordre à courte distance". Ces fluctuations sont observées à toutes concentrations. Leur longueur caractéristique $\xi$ diverge au voisinage d'un point critique. Cela s'explique bien si l'on admet que les interactions entre espèces chimiques sont essentiellement locales : le système appartient alors à la classe d'universalité d'Ising. Cependant, comme les atomes dans les systèmes étudiés occupent les sites d'un réseau cristallin, ce modèle néglige les effets de taille. Dans le cas où les fluctuations de concentration augmentent, cela aboutit à une interaction effective croissante à longue distance. Cahn et Larché /1;2/ont essayé de tenir compte de cet effet. Ils ont montré que, dans le cas de la démixtion, cela aboutit à abaisser la température de décomposition. Il se crée une région métastable dans le diagramme de phases (ex. : Al-Zn). 
(a)

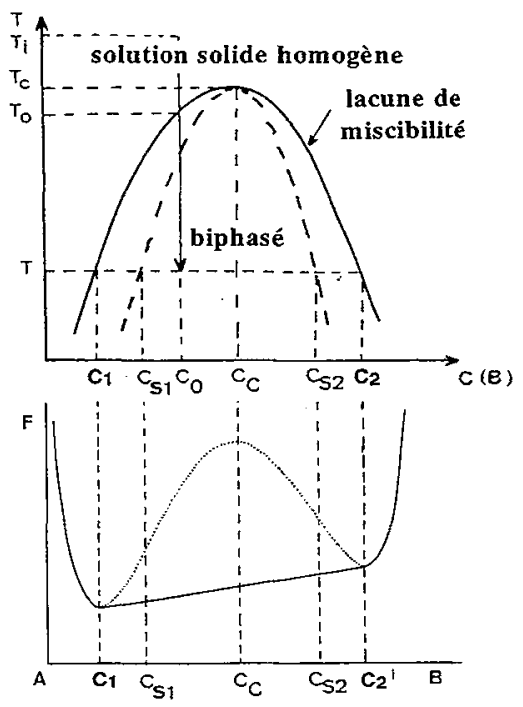

Figure1.- a) Diagramme de phase d'un alliage binaire.

b) Energie libre en fonction de la concentration à la température $T$

Des fluctuations dynamiques de concentration existent également à l'équilibre, dans chacune des phases, lorsque l'alliage est démixté ; leur longueur de corrélation décroît lorsqu'on s'éloigne du point critique.

L'alliage de composition co étant porté instantanément de $\mathrm{T}_{\mathrm{i}}$ à $\mathrm{T}$, l'état de départ de l'évolution contient les fluctuations caractéristiques de la température $T_{\mathfrak{i}}$, ce qui représente un état hautement hors d'équilibre pour la temperature $\mathrm{T}$.

Les théories de la démixtion décrivent la naissance et la croissance de fluctuations de concentration hors d'équilibre qui, à terme, prendront les compositions d'équilibre $c_{1}$ et $c_{2}$. L'expression de l'équation cinétique pour la séparation de phases est donnée par : (voir /3/)

$$
\frac{\partial S(\mathbf{q}, \mathrm{t})}{\partial \mathrm{t}}=-2 \mathrm{Mq} \mathrm{q}^{2}\left[\left(\mathrm{kq}^{2}+\left.\frac{\partial^{2} \mathrm{f}}{\partial \mathrm{c}^{2}}\right|_{c_{0}}\right) \mathrm{S}(\mathbf{q}, \mathrm{t})+\left.\sum_{\mathrm{n}=3}^{\infty} \frac{1}{(\mathrm{n}-1) !} \frac{\partial^{\mathrm{n}} \mathrm{f}}{\partial \mathrm{c}^{\mathrm{n}}}\right|_{c_{0}} \mathrm{~S}_{\mathrm{n}}(\mathbf{q}, \mathrm{t})\right]+2 \mathrm{Mk}_{\mathrm{B}} \mathrm{Tq}^{2}
$$

où $K$ est le coefficient de gradient d'énergie, $M$ la mobilité atomique, et $S_{\mathfrak{n}}(q, t)$ est la transformée de Fourier de la fonction de corrélation à $n$ points $\left(S \equiv S_{2}\right)$.

Un point central pour obtenir la solution de cette équation est de choisir la forme fonctionnelle de l'énergie libre de Helmoltz $\mathrm{f}(\mathrm{c})$. Comme on ne peut résoudre exactement l'équation [1], il faut effectuer des approximations . Deux cas limites sont à considérer : celui de l'apparition de fluctuations à très longue portée et donc avec des gradients de composition faibles (fluctuations homophase), et celui de l'appparition de fluctuations de forte amplitude et localisées (fluctuations hétérophase). Dans le premier cas, il semble raisonnable de retenir simplement les termes lineaires de l'équation et de négliger les termes d'ordre supérieur. Par contre, dans le second cas, il faudra forcément s'en remettre à des équations phénoménologiques, puisqu'il faudrait nécessairement garder les termes à tous les ordres dans l'équation cinétique.

L'équation [1] fait appel à une énergie libre dite de "gros-grains" :

$$
\mathrm{F}\{\mathrm{c}(\mathbf{r})\}=\int \mathrm{dr}\left[\frac{1}{2} \kappa|\nabla c(r)|^{2}+f(c(r))\right] \text {. Conceptuellement, on peut décrire le profil de concentration }
$$
de ces fluctuations, en effectuant une moyenne sur ces "gros-grains", dont la taille doit être inférieure ou de l'ordre de grandeur de $\xi$. Ils sont censés contenir un nombre suffisant d'atomes, pour permettre d'évaluer les grandeurs thermodynamiques nécessaires. Si on écrit l'énergie libre de Helmholtz par unité de volume, d'une solution solide homogene de composition $c$, à la temperature $T_{\mathrm{r}}, f(c), / 4 /$ on obtient (Fig. 1b) une courbe avec deux points d'inflexion $\left(\partial^{2} \mathrm{f} / \partial \mathrm{c}^{2}=0\right)$ qui sont situés sur la courbe spinodale. La spinodale 
sépare la lacune en deux régions : dans la région intérieure, qui correspond au $1^{\mathrm{er}}$ cas, la solution solide est instable même vis à vis de fluctuations de faible amplitude ; dans l'autre région (2 ${ }^{\text {eme }}$ cas), elle est métastable, seules les fluctuations de grande amplitude et de taille supérieure à une taille critique peuvent crôitre. Dans leur étude sur la germination, Cahn et Hilliard /4/ ont montré que lorsqu'on se rapproche de la spinodale, la théorie classique de la germination ne s'applique plus, et que le germe devient de plus en plus diffus, excluant toute discontinuité a la spinodale.

Cette approche est dérivée du champ moyen, et en toute rigueur est valable lorsque la portée des interactions est infinie $15 ; 6 /$. Le domaine de validité est : assez près du point critique pour que la taille des fluctuations soit grande devant celle des atomes, mais pas trop près pour que le champ moyen soit encore valide. Elle s'applique en principe assez difficilement aux alliages métalliques qui ont des interactions à courte portée. Cependant on peut l'utiliser comme un mode de représentation

\section{Premiers stades de démixtion}

\section{- Mode "spinodal"}

Dans le cas où la solution solide est instable, l'approximation linéaire a été proposée par Cahn, Hilliard, Cook $/ 4 ; 7 ; 8 /$.Une question à laquelle il est important de répondre est : quel est le temps pendant lequel les termes suivants, donc non linéaires, peuvent être négligés devant $\left(\mathrm{kq}^{2}+\left.\frac{\partial^{2} \mathrm{f}}{\partial \mathrm{c}^{2}}\right|_{\mathrm{c}_{0}}\right) \mathrm{S}(\mathbf{q}, \mathrm{t})$ ?

Or, ceci revient à se demander si une théorie de champ moyen peut décrire le système au cours de la démixtion. Binder /9/a suggéré une généralisation au cas de la décomposition spinodale du critère de Ginzburg /10/ pour l'applicabilité de l'approximation de champ moyen (les fluctuations du paramètre d'ordre $\left(\mathrm{c}-\mathrm{c}_{0}\right)$ moyenné sur une cellule gros grains, doivent être petites devant le paramètre d'ordre luimême). On trouve /11/ que le temps maximum de validité d'une théorie linéaire est proportionnel au logarithme de la portée de l'interaction ou, selon un paramètre sans dimension, $\varepsilon$, introduit par ces auteurs, à $-\operatorname{Ln}(\varepsilon)$. Dans les systèmes métalliques, où l'interaction est à courte portée, ce temps d'application est en général négatif.

Afin de pouvoir tenir compte des effets des premiers termes non linéaires dans l'équation [1], Langer, Bar-on et Miller /12/ (LBM) ont suggéré une approximation pour la distibution des fluctuations de concentration au cours de la démixtion, qui permet d'introduire un "couplage à champ moyen" entre les modes de fluctuation ; en d'autres termes, les corrélations à $n>2$ corps sont écrites en fonction des corrélations de paire. Le terme de couplage A, qui remplace $\left.\frac{\partial^{2} f}{\partial c^{2}}\right|_{c_{0}}$ dans l'équation linéaire de CHC, est alors donné par : $\quad \mathrm{A}=\left.\sum_{\mathrm{n}=2}^{\infty} \frac{1}{(\mathrm{n}-1) !} \frac{\partial^{\mathrm{n}} \mathrm{f}}{\partial \mathrm{c}^{\mathrm{n}}}\right|_{\mathrm{c}_{0}} \frac{\left\langle(\delta \mathrm{c})^{\mathrm{n}}\right\rangle}{\left\langle(\delta \mathrm{c})^{2}\right\rangle}$ où $\delta \mathrm{c} \equiv \mathrm{c}$-co,, et $<>$ dénote une moyenne pondérée par l'ensemble de distribution de $\delta c$. Pour obtenir une expression simple, il suffit alors d'utiliser une forme telle que $\frac{r}{2}(\delta c)^{2}+\frac{u}{4}(\delta c)^{4}$ avec $r<0$ et $u>0$ pour exprimer $f(\delta c)$.

\section{- germination-croissance}

Dans le cas où la solution solide est métastable, il se crée une barrière d'énergie libre s'opposant au développement de "gouttelettes" de la phase stable. On écrit l’énergie libre d'un précipité de taille r:

$$
F(r)=-A^{3}+B r^{2} \text {, où } A \text { correspond à une énergie libre par unité de }
$$
volume et $\mathrm{B}$ à une énergie libre de surface.

Dans ce cas, correspondant à la germination, on peut, d'après la formule de Gibbs-Thomson,définir un

$$
\text { rayon critique : } \quad r_{c} \approx \frac{2 \sigma_{S} V_{m}}{R T L n \frac{c}{c_{\infty}}}
$$

La probabilite de germination se définit comme la probabilité de constituer une gouttelette de rayon $r \geq$ $r_{c}$, à partir des fluctuations statistiques de la solution solide. La croissance d'une gouttelette isolée dans un milieu sursaturé est un processus de diffusion irréversible dans le gradient de concentration créé au voisinage de la surface. Cette croissance est limitée seulement par la vitesse de diffusion de l'espèce chimique. Dans cette approximation,

$$
\mathbf{r}^{2} \propto \mathrm{Dt} \quad / 13 /
$$


Ce régime n'est jamais observé expérimentalement. Au début de la croissance des premiers germes, d'autres germes se créent concurremment dans la région sursaturée. De plus, on passe très rapidement à un état où la croissance se fait aussi par la disparition de petits précipités (début de la coalescence). Langer et Schwartz (LS) /14/ ont essayé d'étudier la combinaison de ces processus, en fonction de la sursaturation initiale de la solution solide. Ils ont montré que dans le cas d'une faible sursaturation, un processus transitoire complexe séparait l'apparition des premiers germes du régime de coalescence, observé après un temps de plus en plus long lorsque la sursaturation est plus faible, avec une échelle de temps qui peut s'étaler sur 8 ordres de grandeur. Qualitativement, la croissance des précipités et l'appauvrissement de la solution solide sont d'abord accélérés, puis fortement ralentis par rapport au processus observé avec une forte sursaturation (voir ci-dessous l'exemple de $\mathrm{Al}-\mathrm{Li}$ ).

\section{Coalescence}

Lorsque l'essentiel de la solution est décomposée s'établit un processus de "mûrissement d'Ostwald" : les précipités, de taille moyenne $r(t)$ sont en équilibre avec la solution solide restante $: r(t) \approx \frac{2 \sigma_{s} V_{m}}{R T L n \frac{c(t)}{c_{\infty}}}$

Le processus de grossissement est maintenant celui de la diminution des interfaces. Il se produit par "Evaporation" de gouttelettes de petit rayon (inférieur au rayon moyen ci-dessus) et condensation sur les plus grosses. Ce processus, appelé aussi coalescence a été décrit dans le cas où la fraction volumique $\mathrm{f}_{\mathrm{V}}$ des gouttelettes est faible /13; $15 /: \quad r^{3}(t)=K t$

Dans le cas où $f_{\mathrm{v}}$ augmente, on montre que cette équation nécessite l'introduction d'une constante $K$ effective dépendant de $\mathrm{f}_{\mathrm{v}} / 16 /$

\section{Les simulations numériques}

Elles permettent de tester la validité des approximations ci-dessus utilisées. Il s'agit tout d'abord de vérifier à partir de quelle taille l'énergie libre d'un amas s'écrit avec un terme de volume et un terme de surface. Perini, Jacucci et Martin /17/ ont montré que, si l'on est suffisamment loin du point critique, cette approximation est valable à partir d'une dizaine d'atomes.

Il s'agit ensuite de reproduire, par simulation de Monte-Carlo, l'évolution cinétique d'un système. Par exemple $/ 18 /$ il a été possible de montrer, en utilisant les potentiels d'interaction de paires correspondant à $\mathrm{Al}_{3} \mathrm{Li}$, que de petits précipités observés à l'ambiante devenait instables (sous-critiques) lorsque l'alliage est chauffé, même à une température inférieure à la température de décomposition.

L'approximation linéaire de la décomposition spinodale a été vérifiée pendant des temps très courts sur des systêmes où les interactions sont à moyenne portée /19/. La théorie non-linéaire de LBM a été vérifiée $/ 20 /$ en utilisant des interactions de premiers voisins. Cela a surtout servi à essayer didentifier des "courbes universelles" que l'on a comparé à la DPA /5/. Lles simulations vérifient le comportement LSW à temps long. Il reste néanmoins de nombreuses interrogations lorsque les systèmes sont bipercolés.

\section{Méthodes de caractérisation et d'étude}

Il est très important d'utiliser des techniques complémentaires pour bien caractériser les différents stades de la démixtion. La microscopie électronique en transmission ou la microscopie ionique, permettent de visualiser l'espace direct; ces techniques donnent de bonnes informations sur la morphologie des phases ; l'interprétation quantitative des mesures de taille ou de fraction volumique est parfois délicate car la mesure est locale. La diffusion aux petits angles des rayons $\mathbf{X}$ ou des neutrons permet d'obtenir des mesures des dimensions caractéristiques et de l'avancement de la démixtion, mais moyennées sur tout l'échantillon ; il est préférable de connaître par ailleurs la morphologie des phases, pour pouvoir interpréter quantitativement les mesures. D'autres types de mesure permettent d'avoir des informations soit sur la composition des phases, soit sur l'avancement de la démixtion, comme l'effet Mössbauer, la résistivité électrique, la calorimétrie, les propriétés magnétiques. La sonde atomique permet d'obtenir à la fois une mesure de la composition des phases et des dimensions caractéristiques, si l'échantillonage est fait sur un nombre suffisant d'atomes. Les potentialités de cette technique seront décrites par Blavette.

Nous nous attarderons plus ici sur les grandeurs qu'il est possible de mesurer en diffusion aux petits angles.

\section{Diffusion aux petits angles des $\mathrm{RX}$ ou des neutrons}

Dans un alliage binaire $\mathrm{AB}$, complètement désordonné, il existe une diffusion en dehors des raies de Bragg, appelée diffusion de Laue. Elle est constante dans l'espace réciproque, et dépend du "contraste des atomes. En appelant $f_{i}$ les facteurs de diffusion des atomes aux $R X$, ou leurs longueurs de diffusion aux neutrons:

$$
\text { I Laue } \propto c_{A} c_{B}\left(f_{A}-f_{B}\right)^{2}=c_{A} c_{B} \Delta f^{2}
$$




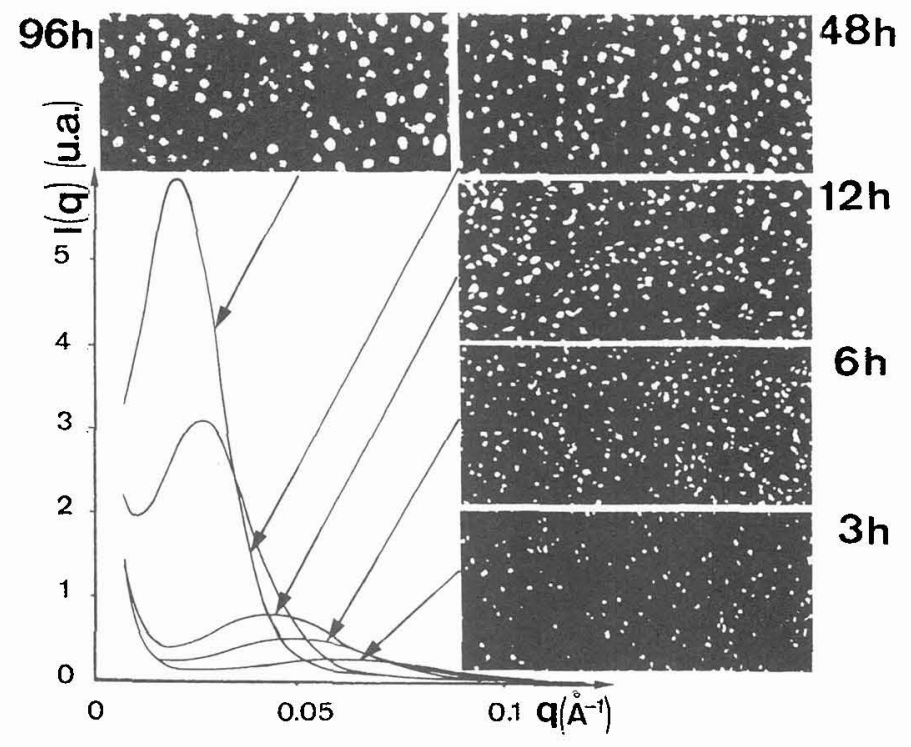

Figure 2.-Evolution de précipités de la phase $\delta$ ' d'un alliage $\mathrm{Al}-8.4 \mathrm{at} \% \mathrm{Li}$ revenu à $150^{\circ} \mathrm{C}$, par DPA de RX in situ, et par microscopie électronique

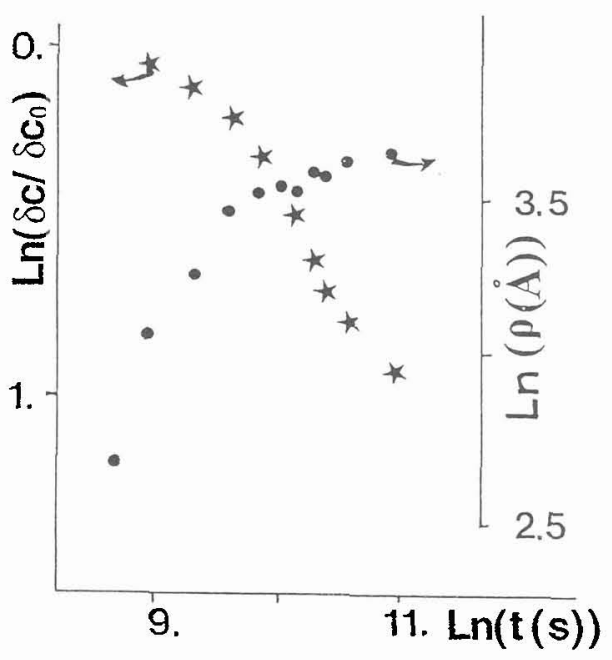

(a)

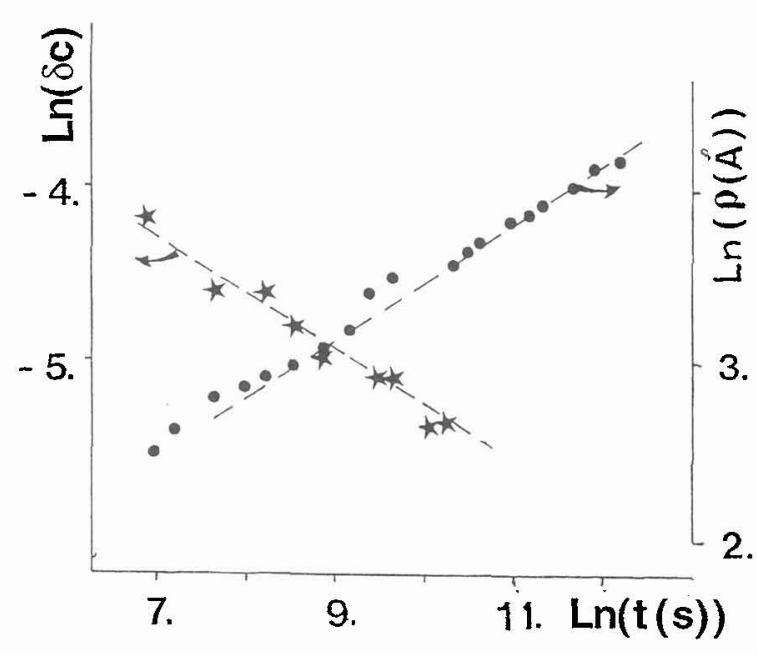

(b)

Figure 3.-Evolution cinétique de la sursaturation de la matrice, et de la taille des précipités, dans $\mathrm{Al}-\mathrm{Li}$ revenu à $150^{\circ} \mathrm{C}$

a) alliage à faible sursaturation $\mathrm{Al}-7.5 \mathrm{at} \% \mathrm{Li}$ b) alliage chargé $\mathrm{Al}-10.5 \mathrm{at} \% \mathrm{Li}$ 
La diffusion diffuse (DD) et la diffusion aux petits angles (DPA) sont des modulations de $\mathrm{I}_{\text {Laue }}$ dues à ce que l'alliage n'est pas complètement désordonné, et donc présente des fluctuations de concentration. Lorsque la longueur d'onde des fluctuations est grande devant la distance interatomique, ce qui est le cas pour une démixtion, cette modulation se produit près des raies de Bragg et du faisceau direct. Cette intensité est proportionnelle à la transformée de Fourier de la fonction de corrélation de concentration :

$$
\mathrm{I}(\mathbf{q}, \mathbf{t}) \propto \Delta \mathbf{f}^{2} \int<\mathrm{c}(\mathbf{r}, \mathbf{t}) \mathrm{c}(\mathbf{r}+\mathbf{R}, \mathbf{t})>\exp (\mathbf{i q} \mathbf{R}) \mathrm{d} \mathbf{R}
$$

Pour obtenir le plus de renseignements quantitatifs il est préférable de normaliser l'intensité pour obtenir des intensités absolues.

Il existe deux approches pour étudier la DPA. Dans la première, comme en diffusion diffuse, on considère la totalité de l'intensité, qui reflète toutes les tailles de fluctuations ; la fonction qui intervient dans les modeles de type CHC ou LBM est directement proportionnelle a I $(q, t)$.

La deuxième approche est plus classique en DPA, elle s'applique mieux quand les deux phases ont chacune une concentration déterminée; on ne doit prendre en compte que la partie proprement petits angles de l'intensité située au dessus de l'intensité diffuse due au désordre interne à chacune des phases.

L'intensité dépend du contraste des deux phases $\Delta \rho$, dans lequel on doit faire intervenir le volume atomique de chaque phase s'ils sont différents (effet élastique). Elle dépend aussi de la forme des précipités, et d'une fonction d'interférence $J(q)$ qui représente les corrélations entre précipités.

$$
\mathrm{I}(\mathrm{q}) \propto \Delta \mathrm{p}^{2} \mathrm{~S}_{\mathrm{pp}}(\mathrm{q})(1-\mathrm{J}(\mathrm{q}))
$$

où $S_{\mathrm{pp}}(q)$ est la fonction de Patterson de la forme des précipités.

Dans chaque phase $j \quad \rho_{j}=\left(c_{A} f_{A}+c_{B} f_{B}\right) / v_{j}, \quad, v_{j}:$ volume atomique dans la phase $j$.

Les longueurs caractéristiques du système qu'on veut en général mesurer sont la dimension des particules et leur espacement. Souvent, on mesure la première par le rayon de Guinier $/ 21$, et la deuxième par la position du pic d'interférence. Il faut comprendre que ces deux mesures se font au niveau du pic d'interférence et que les deux effets s'influencent mutuellement $/ 21 ; 22 \%$. Dans les systèmes concentrés, on obtient une meilleure estimation de la taille des particules avec le rayon de Porod $/ 22 /$, obtenu par la forme du comportement asymptotique de l'intesité $I(q) q \rightarrow \infty=A_{p} q^{-4}+I_{\text {dif }}$

$\mathrm{La}$ recherche de ce comportement asymptotique permet d'évaluer la contribution $\mathrm{I}_{\text {dif }}$ et d'avoir un prolongement analytique, pour éviter les troncatures dans le calcul de fonctions intégrales. Une grandeur intégrale très importante est l'intensité intégrée : (mesures sur un polycristal non texturé)

$$
\mathrm{Q}=\int\left(\mathrm{I}(\mathrm{q})-\mathrm{I}_{\text {dif }}\right) \mathrm{q}^{2} \mathrm{dq} \propto \Delta \mathrm{f}^{2}<\left(\mathrm{c}-\mathrm{c}_{0}\right)^{2}>
$$

Dans un modèle à deux phases $Q \propto \Delta \mathrm{f}^{2} \mathrm{f}_{\mathrm{v}}\left(1-\mathrm{f}_{\mathrm{v}}\right)\left(\mathrm{c}_{1}-\mathrm{c}_{2}\right)^{2}=\Delta \mathrm{f}^{2}\left(\mathrm{c}_{1}-\mathrm{c}_{0}\right)\left(\mathrm{c}_{0}-\mathrm{c}_{2}\right)$

où $f_{v}$ est la fraction volumique de la phase $1, c_{1}$ et $c_{2}$ les concentrations des phases 1 et 2 . La valeur de $Q$ peut donc se relier à l'avancement de la réaction, et dans un système à deux phases à la fraction volumique précipitée.

\section{Exemples de résultats expérimentaux}

\section{Premiers stades de démixtion}

L'étude des premiers stades peut être assez difficile. Pour pouvoir y accéder il faut que l'on puisse retenir par la trempe un état suffisamment désordonné, et donc que la vitesse du changement de température soit assez grande devant la vitesse de la réaction. Il faut trouver un compromis pour limiter la quantité de lacunes retenues en sursaturation pendant la trempe.

\section{- germination-croissance}

En général, le mode germination-croissance est atteint dans ce qu'on appelle des trempes peu profondes, c'est à dire vers un point situé près de la lacune de miscibilité. La germination classique est en général accompagnée du grossissement des germes, et souvent la coalescence commence bien avant la fin de la réaction. Lorsque ce point se rapproche de la lacune, la barrière d'activation pour la nucléation augmente, et on observe un temps d'incubation $/ 23 ; 24 \%$. Lorsqu'on s'en éloigne, le temps d'incubation diminue, et on peut définir une courbe de rosée, qui va dépendre de la sensibilité de la mesure.

- Un système qui illustre la théorie de nucléation-croissance coalescence : Al-Li $/ 25 ; 26 /$

Ce système est un système à mise en ordre, et on observe la décomposition entre la matrice CFC riche en $\mathrm{Al}$ et des précipités $\mathrm{Al}_{3} \mathrm{Li}$ de structure cubique $\mathrm{L}_{2}$. $\mathrm{La}$ fraction volumique de ces précipités peut atteindre $40 \%$, sans qu'il y ait percolation. Comme la transition est de premier ordre, on peut considérer que l'énergie d'interface précipité-matrice reste constante (de 10 à $13 \mathrm{~mJ} / \mathrm{m}^{2}$ ). Enfin les effets élastiques sont faibles et on observe en microscopie électronique que les précipités gardent une forme sphérique pour une large gamme de rayons (de 2 a $100 \mathrm{~nm}$ ) Fig. $2 / 27 \%$.

Dans un alliage à faible sursaturation (Al-7.5at\%Li à $150^{\circ} \mathrm{C}$ ), étudié par DPA des RX, on observe un temps de latence de l'ordre de l'heure où aucune intensité mesurable n'est obtenue. Comme la probabilité de 


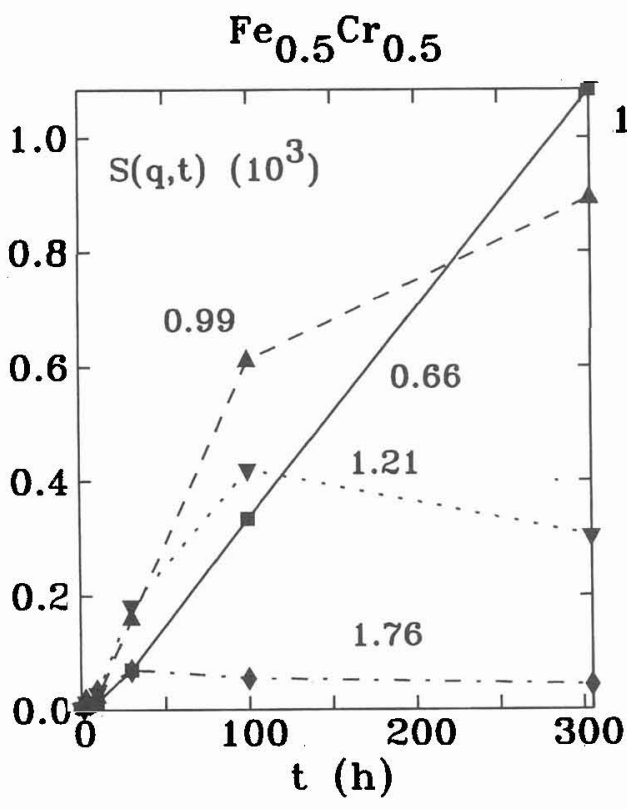

(a)

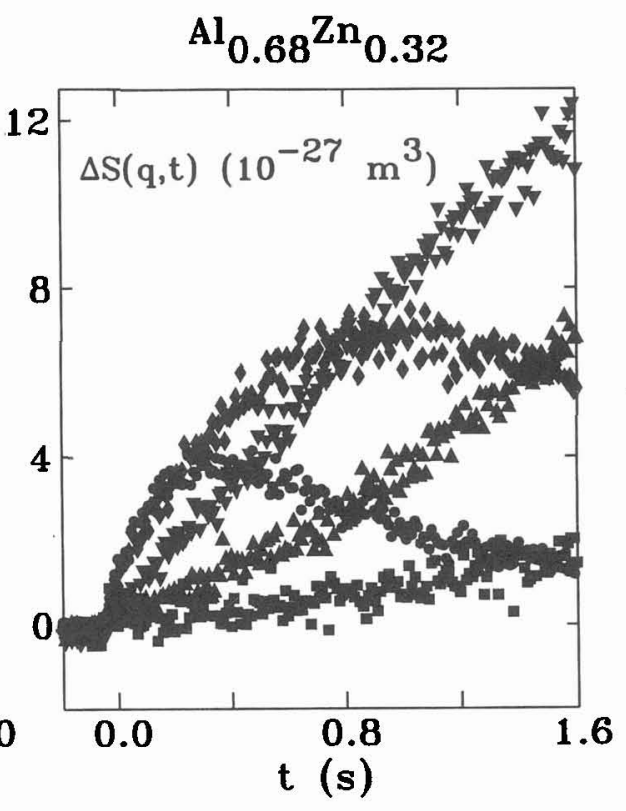

(b)

Figure 4.- Evolution cinétique de $S(q)$ pour quelques valeurs de $q$ a) $\mathrm{Fe}-\mathrm{Cr}$ recuit à $773 \mathrm{~K}$; q en $\mathrm{nm}^{-1}$

b)Al-Zn recuit à $541 \mathrm{~K}\left(0.9 \mathrm{~T}_{\mathrm{c}}\right) ; \square 0.15, \Delta 0.25, \nabla 0.35, \bullet 0.45, \bullet 0.55 \mathrm{~nm}^{-1}$

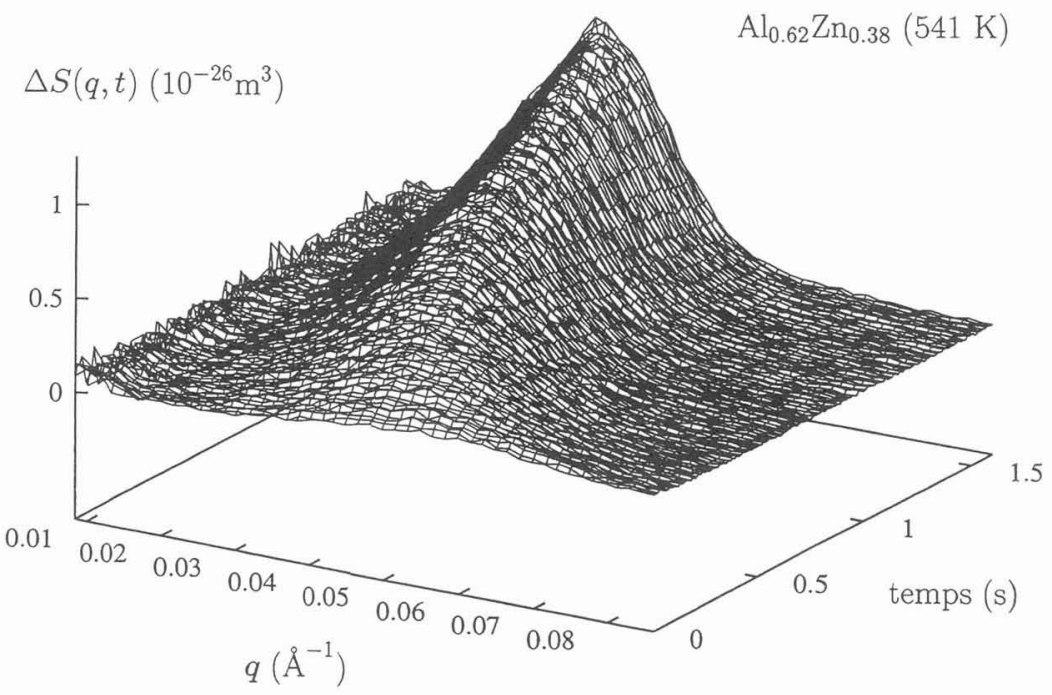

Figure 5.-Evolution cinétique, dans les tous premiers stades, de l'intensité de DPA aux $\mathrm{RX}$, en temps résolu, de $\mathrm{Al}-\mathrm{Zn}$. 
germination est faible, les précipités qui apparaissent croissent ensuite très vite $/ 14 /$. Ce processus est suivi d'un ralentissement lorsque l'essentiel du Lithium en sursaturation a été incorporé dans les précipités. Les courbes se comparent bien aux calculs de L.S. . La Fig. 3-a montre l'évolution de la taille des précipités et de la sursaturation de la matrice dans un alliage peu chargé.

Dans un alliage chargé (Fig.3-b), aucun temps d'incubation n'est mesuré, et on observe immédiatement la coalescence par évaporation-condensation de LSW. Dans ce dernier cas, on peut évaluer le rayon critique à $r_{c} \approx 3 \AA$. Cela veut dire que la barrière de germination est devenue ici négligeable et cela explique que l'on n'observe que la coalescence. Le même type de conclusions a été obtenu dans ce système par /28/

De manière tout à fait équivalente, dans $\mathrm{Cu}-2.7 \mathrm{at} \% \mathrm{Co}$ et $\mathrm{Cu}-1 \mathrm{at} \% \mathrm{Co} / 29 \%$, les premiers précipités mesurables sont presque du Co pur, mais les rayons critiques sont très petits (2-3 $\AA$.

Par ailleurs les théories de germination-croissance $/ 14 /, 130 ; 31 /$ ont été testées dans un certain nombre de systèmes ; elles s'appliquent qualitativement bien pour :

$\mathrm{Al}-6.8 \mathrm{at} \% \mathrm{Zn}$ à $293 \mathrm{~K}$ et $406 \mathrm{~K}, 132 /$

$\mathrm{Fe}-20 \mathrm{at} \% \mathrm{Cr}$ à $773 \mathrm{~K} / 33 /$

$\mathrm{Ni}-36 \mathrm{at} \% \mathrm{Cu}-9 \mathrm{at} \% \mathrm{Al}$ entre 773 et $853 \mathrm{~K} / 34 /$ où les auteurs ont pu déterminer l'énergie d'interface.

$\mathrm{Ni}-14 \mathrm{at} \% \mathrm{Al}$ à $823 \mathrm{~K} / 35$ / où les auteurs ont montré que la coalescence entre en compétition avec la nucléation dès le début.

Souvent,l'application quantitative est assez délicate, car elle nécessite la connaissance de grandeurs thermodynamiques pas toujours évidentes à évaluer.

- Mode "spinodal"

Malgré l'abondance des travaux théoriques sur la décomposition spinodale, il a été exceptionnellement difficile d'obtenir des résultats expérimentaux, en particulier sur les systèmes métalliques. Beaucoup d'efforts ont porté sur la vérification de la théorie linéaire de décomposition spinodale. Néanmoins, à la lumière des connaissances actuelles, il n'existe pas de vérification expérimentale acceptée "sans conditions" de cette théorie, dans un contexte de transition de phase, pour un système métallique. Pour les alliages Fe$\mathrm{Cr}$ et $\mathrm{Fe}-\mathrm{Cr}$-Co situés au centre de la lacune, le mécanisme spinodal semble clairement établi. Dans $\mathrm{Fe}-\mathrm{Cr}$ 136/ ont appliqué le formalisme complet de LBM, et ont pu suivre l'évolution de l'amplitude des fluctuations au cours du temps; $/ 37 /$ et $/ 33 /$ de manière indépendante, ont calculé les coefficients $\mathrm{M}, \mathrm{A}, \mathrm{K}$, et ont trouvé que $A$ a bien l'allure proposée par LBM, sauf aux premiers instants où $A$ est $>0$, ce qui reflète probablement un réajustement des fluctuations, peut-être lié à la trempe. Dans les $\mathrm{Fe}-\mathrm{Cr}$-Co $/ 38 / \mathrm{se}$ sont attachés à montrer, par sonde atomique, l'existence de fluctuations de concentration dans les premiers stades.

Parmi les mesures récentes, mentionnons deux alliages : $\mathrm{Fe}_{0.5} \mathrm{Cr}_{0.5} / 33 /$ et $\mathrm{Al}_{0.62} \mathrm{Zn}_{0.38} / 39 ; 40 /$. Pour ces deux alliages, la Fig. 4 montre bien le comportement non linéaire au cours des premiers stades mesurés de la démixtion : en particulier, on voit que pour certains vecteurs d'onde la courbure change de signe avec le temps. Sur la Fig. 5, on peut voir l'évolution cinétique, en temps résolu de l'alliage de composition critique $\mathrm{Al}_{0.62} \mathrm{Zn}_{0.38}$, mesuré en $\mathrm{RX}$ (synchrotron-NSLS).Dans cet alliage, une analyse suivant l'équation de LBM a permis d'obtenir une valeur pour le paramètre $\varepsilon$ introduit dans la partie théorique, de $\varepsilon=$ $4.84 \pm 0.01$ pour une trempe à $0.9 \mathrm{~T}_{\mathrm{c}}$. Ainsi le temps maximal au cours duquel une théorie linéaire s'appliquerait à la description des premiers stades de la démixtion dans cet alliage serait négatif.

Au cours des stades plus avancés de la démixtion, lorsque des domaines des deux compositions à l'équilibre coexistent et que la cinétique consiste en une croissance de ces domaines, on s'attend à ce qu'une loi d'échelle en $t^{1 / 3}$ soit suivie. Comme on le verra plus loin, la plupart des mesures expérimentales ont donné des exposants inférieurs à $1 / 3$.

\section{Coalescence}

La coalescence est caractérisée par un processus stationnaire : une unique longueur caractéristique $\mathrm{L}$ suffit pour décrire l'évolution du système. En DPA, on peut renormaliser les courbes d'intensité de la manière suivante : $\quad \mathrm{I}(\mathrm{q}) \propto \mathrm{L}^{3} \phi(\mathrm{qL})$ où $\phi(x)$ est une fonction caractéristique du système. Sa forme précise dépend du coefficient qu'on prend pour normaliser les intensités : $I_{\max }$, ou l'intensité intégrée. Plusieurs auteurs ont cherché à donner une forme universelle pour $\phi / 41 ; 42 /$. Qualitativement, $\phi(x)$ a toujours un pic, expliqué par l'interférence entre les précipités. Dans la gamme des faibles fractions volumiques, ce pic s'élargit beaucoup lorsque $\mathrm{f}_{\mathrm{V}}$ décroit comme cela a été observé dans $\mathrm{Al}-\mathrm{Ag}$./43/ Pour les fortes fractions volumiques, $\left(f_{\mathrm{v}} \geq 0.15-0.20\right)$ sa largeur devient plus stable, mais dépend de la morphologie percolée ou non du système $/ 42 / / 26 /$. Le fait que ces courbes soient invariantes, n'est pas, à l'heure actuelle, une preuve formelle du fait qu'une seule longueur soit pertinente, car elles n'y sont pas très sensibles. 
Dans les systèmes à faible sursaturation, on a vu qu'il est difficile d'être sûr d'un exposant de croissance, c'est à dire d'être dans un régime de coalescence, à moins d'avoir fait des mesures sur une importante gamme de temps.

La plupart des alliages étudiés ont des précipités cohérents métastables, qui peuvent (ou non) être précurseurs de la phase stable. Dans ce cas la phase stable apparaît aux longs temps de vieillissement. Ces phases stables sont souvent semi-cohérentes, voire incohérentes avec la matrice. Elles ont en général une forte énergie d'interface $\left(\geq 100 \mathrm{~mJ} / \mathrm{m}^{2}\right)$, des dislocations d'interface ... Pour cette raison elles ne peuvent se développer directement à partir de la solution solide (trop fort rayon critique). Lorsque la phase stable apparaît, l'évolution de la phase homogène ne peut plus être étudiće!

Dans les alliages non percolés, lorsque l'essentiel de la solution solide est décomposée, le régime stationnaire d'évaporation-condensation de gouttelettes est attendu (LSW). Dans ce cas, le rayon des précipités augmente comme $t^{\mathbf{a}}$ avec $\mathbf{a}=1 / 3$. Cela est observé essentiellement dans des régimes où l'effet de taille dû aux différences de paramètre de maille entre les précipités et la matrice est faible (Ni-Al /35/, Ni$\mathrm{Al}-\mathrm{Mo} / 44 /, \mathrm{Al}-\mathrm{Li} / 25 /$., Fe-Cr-Co à précipités sphériques $/ 38 /$..)

Dans les autres cas, a est inférieur à $1 / 3$, et cela se combine souvent avec une croissance anisotrope des précipités (Al-Cu, $\mathrm{Al}-\mathrm{Zn} / 32 /, \mathrm{Cu}-\mathrm{Ni}-\mathrm{Fe} / 45 ; 46 /$ )

Lorsque les phases sont bipercolées, des valeurs de a $<1 / 3$ ont été trouvées, dans des conditions où le régime de coalescence semble atteint (intensité intégrée constante, "comportement universel") :, $\mathrm{Fe}-\mathrm{Cr}-\mathrm{Co}$ $147 ; 48 /$ et $/ 38 /$ où $a \approx 0.24$ quand l'alliage est bipercolé, alors que $a=1 / 3$, quand les précipités sont sphériques. Dans l'alliage Cu-Mn dans des conditions où on pourrait s'attendre à ce que l'alliage soit bipercole, $/ 49 /$ trouve $\mathbf{a}=1 / 3$. Dans $\mathrm{Al}-\mathrm{Zn}$ à la composition critique, un exposant $\mathbf{a}>0.4 \mathrm{a}$ été trouvé.

$\mathrm{La}$ question qui reste à trancher est $/ 50 /$ de savoir si la valeur de cet exposant a, lorsqu'elle est différente de 1/3 est seulement transitoire ou non, et quel mécanisme intervient, autre que l'évaporation condensation.

\section{Conclusion}

L'ensemble des étapes de démixtion se produisant sur une gamme de temps très importante, il est parfois difficile d'observer tous les stades mis en jeu : processus complexe de germination-croissancecoalescence lors de trempes peu profondes, décomposition spinodale lors de trempes profondes, puis coalescence. Les effets élastiques abaissent la position de la lacune de miscibilité, et affectent la cinétique de coalescence. Enfin l'évolution à très long terme d'alliages à structure bipercolée mériterait d'être approfondie.

\section{Références}

/1/ Cahn, J.W., Acta Metall. 10 (1962) 907

/2/ Cahn, J.W. and Larché, F.C., Acta Metall. 32 (1984) 1915

13/ Gunton, J.D., San Miguel, M. and Sahni, P.S., in Phase Transitions and Critical Phenomena (eds. Lebowitz, C.D.a.J.L.) (Academic Press, London, 1983) 267

/4/ Cahn, J.W. and Hilliard, J.E., J. Chem. Phys. 31 (1959) 688

15/ Binder, K., in Materials Science and Technology : Phase Transformations in Materials (eds.

Haasen, P.) (VCH Verlagsgesellschaft, Weinheim, 1991) 405

/6/ Binder, K. and Stauffer, D., Adv. Phys. 25 (1976) 343

/7/ Cahn, J.W., Acta Metall. 9(1961) 795

/8/ Cook, H.E., Acta Metall. 18 (1970) 297

19/ Binder, K., Phys. Rev. A 29 (1984) 341

/10/ Ginzburg, V.L., Sov. Phys. - Solid State 2 (1960) 1824

/11/ Grant, M., San Miguel, M., Vinals, J. and Gunton, J.D., Phys. Rev. B 31 (1985) 3027

/12/ Langer, J.S., Bar-on, M. and Miller, H.D., Phys. Rev. A 11 (1975) 1417

113/ Wagner, C., Z. Für Elektrochemie 65 (1961) 581

/14/ Langer, J.S. and Schwartz, A.J., Phys. Rev. A 21 (1980) 948

/15/ Lifschitz, I.M. and Slyozov, V.V., J. Phys. Chem. Solids 19 (1961) 35

/16/ Voorhees, P.W. and Glicksman, M.E., Act. Metall. 32 (1984) 2013

/17/ Perini, A., Jacucci, G. and Martin, G., Surface Science 144 (1984) 53

/18/ Livet, F. and Brechet, Y., J. de Phys. 48 -C3 (1987) 357

/19/ Heermann, D.W., Phys. Rev. Lett. 52 (1984) 1126

/20/ Marro, J., Bortz, A.B., Kalos, M.H. and Lebowitz, J.L., Phys. Rev. B 12 (1975) 2000

121/ Guinier, A. and Fournet, G.,Small-angle scattering of X-rays 1 (John Wiley \& Sons, New-York, 1955) 268

122/ Porod, G., in Small Angle X-ray Scattering (eds. Glatter, O. and Kratky, O.) (Academic Press

Inc., London, 1982) 17

/23/ Laslaz, G. and Guyot, P., Acta metall. 25 (1977) 277 
124/ Luiggi, N., Simon, J.P. and Guyot, P., Acta Metall. 28 (1980) 1115

/25/ Livet, F. and Bloch, D., Scripta Met. 19 (1985) 1147

/26/ Bley, F., Guyot, P., Livet, F. and Simon, J.P., Phase Trans. 31 (1991) 201

/27/ Gomiero, P., Thèse de Doctorat, Institut National Polytechnique de Grenoble (France), (1990).

/28/ Schmitz, G. and Haasen, P., Acta metall. mater. 40 (1992) 2209

/29/ Haasen, P. and Piller, J., Z. Metallkde. 78 (1987) 757

130/ Kampmann, R. and Wagner, R., in Decomposition of Alloys: the early stages (eds. Haasen, P.,

Gerold, V., Wagner, R. and Ashby, M.F.) (Pergamon Press, Oxford, 1984) 91

131/ Wagner, R. and Kampmann, R., in Materials Science and Technology : Phase Transformations in

Materials (eds. Haasen, P.) (VCH Verlagsgesellschaft, Weinheim, 1991) 213

/32/ Simon, J.P., Guyot, P. and Salva, A.D., Phil. Mag. A49 (1984) 151

133/ Bley, F., Acta metall. mater. 40 (1992) 1505

134/ Liu, Z.G. and Wagner, R., J. de Phys. France 45 - C9 (1984) 441

135/ Wendt, H. and Haasen, P., Acta Metall. 31 (1983) 1649

/36/ La Salle, J.C. and Schwartz, L.H., Acta metall. 34 (1986) 989

137/ Furusaka, M., Ishikawa, Y., Yamaguchi, S. and Fujino, Y., J. Phys. Soc. Jpn. 55 (1986) 2253

138/ Zhu, F., Haasen, P. and Wagner, R., Acta metall. 34 (1986) 457

139/ Mainville, J., Yang, Y.S., Elder, K.R., Sutton, M., Ludwig Jr., K.F. and Stephenson, G.B., Soumis à Phys. Rev. Lett.

140/ Mainville, J., Ph.D. Thesis, Center for the Physics of Materials, Department of Physics,McGill University, Canada, (1992).

/41/ Furukawa, H., J. Phys. Soc. Jap. 58 (1989) 216

142/ Fratzl, P. and Lebowitz , J.L., Acta metall. 37 (1989) 3245

143/ Langmayr, F., Fratzl, P. and Vogl, G., Acta metall. mater. 40 (1992) 3381

144/ Calderon, H. and Kostorz, G., Mat. Res. Soc. Symp. Proc. 166 (1990) 255

145/ Lyon, O. and Simon, J.P., Phys. Rev. B35 (1987) 5164 1905

146/ Poerschke, R., Wagner, W., Wollenberger, H. and Fratzl, P., J. Phys. F: Met. Phys. 16 (1986)

/47/ Brenner, S.S., Camus, P.P., Miller, M.K. and Soffa, W.A., Acta metall. 32 (1984) 1217

148/ Simon, J.P. and Lyon, O., Act. Metall. 37 (1989) 1727

/49/ Gaulin, B.D., Spooner, S. and Morii, Y., Phys. Rev. Letters 59 (1987) 668

150/ Simon, J.P., Guyot, P. and Lyon, O., Solid State Phenom. 3\&4 (1988) 179 